\title{
Schader-Preis 2013 für Jutta Allmendinger
}

Die Soziologin Jutta Allmendinger erhält den Schader-Preis 2013. Mit dem Preis zeichnet die Schader-Stiftung Gesellschaftswissenschaftlerinnen und -wissenschaftler aus, die durch ihre wissenschaftliche Arbeit und ihr öffentliches Wirken wichtige Beiträge für die Lösung gesellschaftlicher Probleme geleistet haben.

Jutta Allmendinger steht mit ihren vielfältigen öffentlichkeitswirksamen Arbeiten für die konsequente Vermittlung sozialwissenschaftlicher Forschungsthemen und -ergebnisse in die Öffentlichkeit. Seit 2007 ist sie Präsidentin des Wissenschaftszentrums Berlin für Sozialforschung (WZB). Untersucht werden dort, aus Perspektiven verschiedener Disziplinen, zentrale gesellschaftliche Themen: Armut, politische und gesellschaftliche Teilhabe, Arbeit, Integration, internationale Konflikte, Innovation und Wettbewerb. Jutta Allmendinger selbst hat ihre Arbeitsmarkt- und Bildungsforschung mit dem Thema Ungleichheit der Lebenschancen sowie Geschlechter- und Familienfragen verknüpft und dabei immer den internationalen Vergleich einbezogen.

Jutta Allmendinger ist eine außergewöhnliche Persönlichkeit, die als Präsidentin des WZB und Professorin für Bildungssoziologie und Arbeitsmarktforschung an der Humboldt-Universität zu Berlin Theorie und Praxisbezogenheit bestens verbindet.

Der Schader-Preis wird jährlich von der Schader-Stiftung verliehen und ist mit $15.000 €$ dotiert. Preisgericht ist das Kuratorium der Stiftung. Zu den bisherigen Preisträgern zählen unter anderem Paul Kirchhof (2012), Jan Philipp Reemtsma (2011), Wolf Lepenies (2010), Lord Ralf Dahrendorf (2009), Klaus von Beyme (2008), Franz-Xaver Kaufmann (2007), Gesine Schwan (2006) und Ulrich Beck (2005). Die Schader-Stiftung fördert die Gesellschaftswissenschaften und deren Dialog mit der Praxis.

(c) Springer Fachmedien Wiesbaden 2013 